\title{
Management practices for asparaginase-associated coagulopathy: a survey of pediatric oncologists
}

\author{
Jacob Greenmyer ${ }^{1}$, Kirk Wyatt ${ }^{2}$, Vilmarie Rodriguez ${ }^{3}$, Aneel Ashrani ${ }^{1}$, and Deepti Warad ${ }^{1}$ \\ ${ }^{1}$ Mayo Clinic \\ ${ }^{2}$ Sanford Health \\ ${ }^{3}$ Nationwide Children's Hospital
}

July 19, 2021

\begin{abstract}
BACKGROUND: Coagulopathy and thrombosis are well-described complications of asparaginase therapy; however, treatment practices in pediatric hematology oncology patients vary widely as evidence-based guidelines for clinical management of these complications in this population are lacking. OBJECTIVE: To assess management practices of asparaginase-related coagulopathy by pediatric hematology oncology (PHO) providers. DESIGN/METHOD: Email survey sent to 2,327 PHO providers primarily practicing in the United States. RESULTS: Two hundred and eighty-five $(12.2 \%)$ attending physicians completed the survey. Only $4.6 \%(n=13 / 285)$ routinely prescribe prophylactic anticoagulation during induction chemotherapy for leukemia. Slightly more than half $(n=145 / 250,50.9 \%)$ of all providers perform baseline coagulation studies. Most providers that were surveyed $(n=185 / 285,64.9 \%)$ only replete coagulant factors if the patient experiences bleeding or bruising. One hundred and thirty $(\mathrm{n}=130 / 285,45.6 \%)$ physicians replace low fibrinogen, and the median replacement was $100 \mathrm{mg} / \mathrm{dL}(\mathrm{range}: 40-200$ $\mathrm{mg} / \mathrm{dL}$ ) with the median target of at least $100 \mathrm{mg} / \mathrm{dL}$ (range: 50-200 mg/dL). A minority of physicians $(\mathrm{n}=39 / 250,13.7 \%$ ) replace low antithrombin at a median cutoff activity level of 60\% (range 40-100\%) with a median target of $75 \%$ (range: 40-125\%). CONCLUSION: There is a significant variation in PHO provider practices for monitoring and management of asparaginase-associated hemostatic derangements. Evidence-based guidelines have the potential to standardize practices.
\end{abstract}

\section{INTRODUCTION}

Following its discovery in the 1950's, asparaginase became a cornerstone of pediatric antileukemic therapy, yet it is associated with a number of acute toxicities which contribute to early morbidity in children with leukemia and may adversely impact event-free survival in cases requiring discontinuation of asparaginase (Gupta et al., 2020; Kidd, 1953). By way of depleting the body of the amino acid asparagine, asparaginase leads to decreased production of a number of proteins involved in coagulation. This effect may manifest as either thrombotic or bleeding sequelae (Andrew, Brooker, \& Mitchell, 1994; Hernandez-Espinosa et al., 2006; Hongo et al., 2002; Mitchell, Hoogendoorn, Giles, Vegh, \& Andrew, 1994; L. G. Mitchell et al., 1994). Thrombotic complications are thought to arise from reduced levels of natural anticoagulants such as protein C, protein S, plasminogen, and antithrombin; while bleeding risk is primarily attributed to hypofibrinogenemia (Caruso et al., 2006; Payne \& Vora, 2007).

Data from clinical trials suggest that the incidence of symptomatic thrombosis among patients receiving antileukemic therapy with asparaginase ranges from 2-7\%. (Caruso et al., 2006; Nowak-Gottl, Kenet, \& Mitchell, 2009; Payne \& Vora, 2007; Plourde et al., 2014; Qureshi, Mitchell, Richards, Vora, \& Goulden, 2010; Salzer et al., 2013). A meta-analysis of 17 studies focused on thrombotic complications in children with ALL found that the overall incidence of symptomatic thrombosis was 5.2\% (Caruso et al., 2006). The majority of symptomatic thromboses were reported to occur in the central nervous system (CNS), manifesting most 
commonly as cerebral sinovenous thrombosis. Of the non-CNS events, the greatest incidence was seen in the upper limbs, most commonly manifest as central venous catheter-related deep vein thrombosis (Caruso et al., 2006). The Prophylactic Antithrombin Replacement in Kids with Acute Lymphoblastic Leukemia Treated with Asparaginase (PARKAA) study screened children with leukemia undergoing asparaginase therapy for thrombotic events and observed that over one third (36.7\%) developed venous thrombosis, most commonly in the upper central venous system. Only 3 out of 22 patients with thrombotic events (14\%) presented symptomatically; the remainder were diagnosed on surveillance imaging only. These results suggest that the incidence of venous thrombosis is much higher than initially suspected, with a high burden of asymptomatic thrombosis. In the light of this observation, the Children's Oncology Group study ACCL 1333 is studying the role of apixaban for prevention of asparaginase-associated deep vein thrombosis (O'Brien, 2019).

In contrast to thrombotic complications, the burden of hemorrhagic complications from asparaginase therapy is less clear. In our experience, the most noteworthy complication is the development of spinal epidural hematoma following lumbar puncture (Qubty, Mrelashvili, \& Patterson, 2015). This is relevant because intrathecal therapy is administered frequently throughout antileukemic therapy and the morbidity of this complication can be high.

Expert opinion-based recommendations for prevention of thrombotic and hemorrhagic complications of asparaginase therapy have been published for adults and older adolescents (Aldoss \& Douer, 2020; Stock et al., 2011). Meanwhile, recommendations for pediatric patients are sparse. In the existing literature, prevention and management strategies including antithrombin and fibrinogen replacement therapies are highly variable - in particular, there is a lack of consensus in the triggers used for antithrombin or fibrinogen replacement (Aldoss \& Douer, 2020; Stock et al., 2011). The PARKAA study demonstrated that antithrombin replacement for prevention of thrombotic events was safe and observed a trend toward reducing venous thromboembolism in children during asparaginase therapy, but the difference failed to reach statistical significance (28\% vs. 37\%) (L. Mitchell et al., 2003). Replacement of antithrombin can be challenging due to its relatively short half-life, which demands frequent intravenous infusions, and its cost. A prospective study comparing low-molecular-weight heparin and antithrombin versus antithrombin replacement alone (for a level $<50 \%$ ) for the prevention of symptomatic venous thromboembolism in children with acute lymphoblastic leukemia demonstrated a benefit for combined therapy over antithrombin alone (Meister et al., 2008). However, this study did not identify subpopulations whom would benefit most from prophylaxis and was not designed to assess the benefit of antithrombin therapy alone compared to no therapy (Meister et al., 2008; L. Mitchell et al., 2003).

Due to the lack of of evidence-based recommendations for managing asparaginase-related coagulopathy and variable practices, we aimed to survey practices for management of asparaginase coagulopathy in pediatric patients.

\section{METHODS}

This study was reviewed by the Mayo Clinic Institutional Review Board. Study data were collected using and managed using REDCap electronic data capture tools hosted at Mayo Clinic (Harris et al., 2019; Harris et al., 2009).

Survey invitations were emailed to 2,327 PHO providers (attending physicians and advanced clinical practitioners) who primarily practiced in the United States. Survey questions and the survey instrument were constructed and revised by three practicing pediatric hematologist/oncologists with an interest in asparaginase related coagulation abnormalities. Respondents were able to respond over the course of 2 months. Up to three periodic email reminders were sent to those who had not yet completed the survey. All data was collected in a deidentified manner.

Statistical analysis was performed in REDCap (Version...) and Microsoft Excel (version...). Descriptive statistics such as mean, median, standard deviation, and range were used to describe and summarize survey findings. 


\section{RESULTS}

\section{Participants}

Sample characteristics of respondents are as summarized in Table 1. Two hundred and eighty-five (12.2\%, $\mathrm{n}=285 / 2327)$ attending physicians completed the survey. The largest proportions of responding physicians had practiced for $<5$ years $(28.8 \%, \mathrm{n}=82 / 285)$ or $>20$ years $(23.5 \%, \mathrm{n}=67 / 285)$. A majority of respondents $(64.2 \%, \mathrm{n}=183 / 285)$ individually managed between 10 and 40 patients under the age of 21 years with acute lymphoblastic leukemia annually. Most respondents reported encountering a patient with asparaginase-associated thrombosis $(94.7 \%, \mathrm{n}=270 / 295)$. Asparaginase-related bleeding complications were less frequently encountered, though observed by nearly half of respondents $(45.4 \%, \mathrm{n}=129 / 284)$.

\section{Management of complications}

Only $34 \%$ (97/285) reported the existence of local institutional practice guidelines for managing hemorrhagic or thrombotic complications related to asparaginase (Appendix 1). Twenty-seven percent of respondents (n $=77 / 285$ ) reported no uniformity in local institutional practice for management of bleeding or thrombosis. Recommendations published in the literature are used by $40.7 \%(\mathrm{n}=116 / 285)$ of physicians to manage complications, with the CHEST guidelines (Monagle et al., 2012) $(\mathrm{n}=20 / 60)$ and ASH Guidelines (Ortel et al., 2020) $(\mathrm{n}=11 / 60)$ being the most commonly cited.

\section{Prophylactic anticoagulation}

Only $4.6 \%$ ( $\mathrm{n}=13 / 285)$ routinely prescribe prophylactic anticoagulation during induction chemotherapy for leukemia with asparaginase. Some providers $(4.6 \%, \mathrm{n}=13 / 285)$ reported utilizing prophylactic anticoagulation in specific high-risk patient sub-populations such as patients who are obese/overweight (BMI $>25)$ $(69.2 \%, \mathrm{n}=9 / 13)$, patients receiving four drug induction $(61.5 \%, \mathrm{n}=8 / 13)$, and teenagers/adolescents $(61.5 \%$, $\mathrm{n}=8 / 13$ ) (Appendix 2).

\section{Laboratory testing}

Slightly more than half $(\mathrm{n}=145 / 250,50.9 \%)$ of all providers perform baseline coagulation studies on patients undergoing asparaginase therapy (Appendix 3). Among those who ordered coagulation labs routinely, $41 \%$ $(\mathrm{n}=59 / 144)$ repeat testing only if abnormal, $19.4 \%(\mathrm{n}=28 / 144)$ order labs prior to invasive procedures such as lumbar punctures, and $11.1 \%(\mathrm{n}=16 / 144)$ repeated labs weekly. Commonly ordered baseline laboratory studies include a complete blood count $(\mathrm{n}=90 / 145,62.1 \%)$, PT $(\mathrm{n}=86 / 145,59.3 \%)$, aPTT $(\mathrm{n}=85 / 145$, $58.6 \%)$, fibrinogen $(\mathrm{n}=84 / 145,57.9 \%)$, and antithrombin $(\mathrm{n}=24 / 145,16.6 \%)$.

\section{Pharmacologic replacement}

Most that were surveyed ( $\mathrm{n}=185 / 285,64.9 \%)$ repleted procoagulant factors if the patient experiences bleeding or increased bruising, compared to $48.4 \%$ (138/285) who repleted prior to procedures. One hundred and thirty $(\mathrm{n}=130 / 285,45.6 \%)$ physicians replaced low fibrinogen at a median cutoff level of $100 \mathrm{mg} / \mathrm{dL}$ (range: 40-200) with the median target of at least $100 \mathrm{mg} / \mathrm{dL}$ (range: 50-200) (Figure 1, Appendix 4). The most common replacement products included cryoprecipitate $(\mathrm{n}=110 / 130,84.6 \%)$, fresh frozen plasma $(\mathrm{n}=37 / 130,28.5 \%)$ and fibrinogen concentrate $(\mathrm{n}=21,16.2 \%$, [Appendix 4$])$.

Only $13.7 \%$ ( $\mathrm{n}=39 / 285)$ of those surveyed had a cut off level of low antithrombin below which they correct. Almost all utilized antithrombin concentrate $(97.1 \%, \mathrm{n}=33 / 34)$ instead of fresh frozen plasma $(2.9 \%$, 1/34). Respondents replaced low antithrombin at a median cut off of 60\% (range 40-100\%) and attempted to achieve a median level of at least $75 \%$ (range 40-125\%) $(\mathrm{n}=25)$ (Figure 2, Appendix 5). There were no statistically significant differences in practices of anticoagulation prescription, baseline lab monitoring, or antithrombin replacement practices, based on whether respondents had encountered thrombotic complications of peg-asparaginase in practice (Appendices 6-8). Similarly, there were no statistically significant differences in practices of anticoagulation prescription, baseline lab monitoring, or fibrinogen replacement, based on whether respondents had encountered peg-asparaginase associated bleeding complications in their clinical practice (Appendices 9-11). 


\section{DISCUSSION}

We describe the results of an online survey responded to by 285 pediatric hematologist/oncologists who reported on their practices for monitoring coagulation studies and pharmacologic replacement in children, adolescents, and young adults treated with asparaginase. Routine use of prophylactic anticoagulation in patients receiving asparaginase was rare. While coagulation factor monitoring was reported by half of respondents, there was significant variability in terms of which patients were monitored and how often monitoring took place. Among providers who corrected low fibrinogen, the median replacement trigger for fibrinogen was $100 \mathrm{mg} / \mathrm{dL}$. with a goal level of at least $100 \mathrm{mg} / \mathrm{dL}$. Among providers who corrected low antithrombin, the median replacement trigger was $60 \%$, with a goal level of at least $75 \%$.

Pediatric oncologists are well-aware that thrombotic and hemorrhagic complications can occur in patients receiving asparaginase therapy. However, it is unclear how to best prevent these complications. One rational approach is to monitor and replace of fibrinogen immediately prior to lumbar punctures, yet it remains unclear which patients would benefit from such monitoring and replacement, the timeframe following asparaginase doses during which to monitor, and what level of fibrinogen is necessary. In our study, respondents that monitor for asparaginase coagulopathy do so in average risk ALL patients, high risk ALL patients, obese/overweight patients, and prior to invasive procedures such as lumbar puncture.

Prevention of thrombotic complications has received significant attention (Nowak-Gottl et al., 2009). In addition to anticoagulant factor depletion caused by asparaginase, children with leukemia have additional venous thrombosis risk factors including cancer and central venous catheters. The PARKAA study revealed that symptomatic venous thromboses were just the "tip of the iceberg," as over one third of pediatric patients will experience a thrombotic event while on asparaginase (L. G. Mitchell et al., 2003). The study demonstrated a trend toward benefit for antithrombin replacement for preventing venous thrombosis; however, the observed difference was not statistically significant and antithrombin replacement requires frequent intravenous infusions at significant cost, making the benefit of this practice questionable (L. Mitchell et al., 2003). While the use of low molecular weight heparin has been proposed for prevention of deep vein thrombosis in children undergoing antileukemic therapy, this approach is problematic in patients who are deficient in antithrombin, as heparin is less effective in states of antithrombin deficiency, and it also introduces challenges because of the need to hold the medication around the time of frequent lumbar punctures (Elhasid et al., 2001).

Patients with newly diagnosed acute lymphoblastic leukemia treated in the ALL-BFM 2000 and AIEOPBFM ALL 2009 ( $\mathrm{n}=949$, age 1 to 18 years) were randomized to receive low-dose unfractionated heparin, prophylactic low molecular weight heparin (enoxaparin) or activity-adapted antithrombin throughout induction therapy (Greiner, 2019). Thromboembolism complications occurred in 42 patients (4.4\%). Patients assigned to unfractionated heparin had a higher risk of thromboembolism (8.0\%) compared with those randomized to enoxaparin $(3.5 \% ; P=0.011)$ or antithrombin $(1.9 \% ; P<0.001)$. However, The 5-year event-free survival was $80.9 \pm 2.2 \%$ among patients assigned to antithrombin compared to $85.9 \pm 2.0 \%$ in the unfractionated heparin group $(P=0.06)$, and $86.2 \pm 2.0 \%$ in the enoxaparin group $(P=0.10)$. The findings of lower EFS for those patients receiving antithrombin for thromboprophylaxis remains unclear. The subcutaneous route of administration presents a challenge for administration in young children, and bleeding risk may be complicated by thrombocytopenia, which often arises secondary to chemotherapy. Children's Oncology Group is currently investigating the role of apixaban for deep vein thrombosis prophylaxis in patients undergoing therapy with asparaginase (O'Brien et al., 2019). Benefits of this agent include oral route of administration, anticoagulant effect independent of antithrombin levels and no need for routine laboratory monitoring.

There are several limitations to this study. First, this study was a survey, so it is susceptible to response bias. Respondents may have responded based on clinical exposure to asparagine-related thrombosis or academic interest. Moreover, because this study was anonymous, it was not possible to determine the institutional or geographic distribution of respondents. Indeed, because we did not limit number of responses from individual institutions, the institutional practices of some larger organizations may be overrepresented in survey respondents. Finally, it is important to note that this survey does not represent expert recommendations or consensus criteria, but instead provides an overview of the varied landscape of management practices by 
pediatric hematologists oncologists.

Nonetheless, this study has several strengths. In our knowledge, this is the first study that provides a comprehensive overview of prophylaxis, replacement, and treatment practices of pediatric oncologists who prescribe asparaginase in United States. Secondly, this study clearly demonstrates the variability of management practices and lays the groundwork for future efforts towards establishing consensus guidelines. Moreover, the importance of documenting and publishing current practices was emphasized by multiple respondents who contacted our team to candidly express their desire to review the results of the survey.

Until best-practice approaches for prevention of thrombotic and hemorrhagic complications of asparaginase therapy are established through randomized controlled trials, the optimal approach to prevention of these complications will remain unclear. At Mayo Clinic, we do not monitor or replace antithrombin routinely because this practice is logistically cumbersome and its benefit was not proven in the PARKAA study. We do, however, monitor fibrinogen prior to invasive procedures and replace fibrinogen levels $<100 \mathrm{mg} / \mathrm{dL}$ to a goal of $120 \mathrm{mg} / \mathrm{dL}$ with either fibrinogen concentrate or cryoprecipitate in children 12 years of age or greater and in patients receiving doses of pegaspargase of 3,000 units or greater. The latter criteria were used because patients who are older and who receive higher doses of asparaginase were anecdotally observed to experience frequent thrombotic events as a result of derangements of coagulation factors, and this effect was extrapolated to hemorrhagic complications. We monitor coagulation parameters for four weeks following doses of pegasparaginase based on adult data suggesting that asparagine depletion lasts for up to four weeks (Douer et al., 2007). We do not routinely prescribe prophylactic anticoagulation in patients undergoing antileukemic therapy with asparaginase because there is no preferred prophylactic therapy; however, we consider it on a case-by-case basis for children with additional risk factors (e.g., obesity, bulky lymphadenopathy with compression of blood vessels etc.). Vitamin K antagonists (i.e., warfarin) are challenging to consistently dose in young children undergoing antineoplastic chemotherapy who often experience swings in appetite,dietary intake, and are on multiple drugs with potential for interactions. Low molecular weight heparin (e.g., enoxaparin) requires antithrombin to exert an anticoagulant effect, and antithrombin levels may be insufficient following asparaginase therapy. Furthermore, although a recent phase 3 randomized controlled trial recently reported on the safety and efficacy of rivaroxaban for pediatric deep vein thrombosis, direct acting oral anticoagulants (e.g., apixaban, rivaroxaban) have not yet been proven effective for deep vein thrombosis prophylaxis in children undergoing antileukemic therapy, though we are hopeful that the ACCL 1333 study will inform the safety and efficacy of the use of these agents (Male, 2020).

In summary, our study provides a snapshot of the current practices of PHO providers in USA for management of asparaginase associated coagulopathy in patients aged $<21$ years. To improve the care of children and adolescents undergoing asparaginase based therapy, we reiterate the importance of developing evidence-based consensus guidelines to guide providers regarding best-practices based on the best available evidence.

CONFLICTS OF INTEREST: None.

\section{CITATIONS}

Aldoss, I., \& Douer, D. (2020). How I treat the toxicities of pegasparaginase in adults with acute lymphoblastic leukemia.Blood, 135 (13), 987-995. doi:10.1182/blood.2019002477

Andrew, M., Brooker, L., \& Mitchell, L. (1994). Acquired antithrombin III deficiency secondary to asparaginase therapy in childhood acute lymphoblastic leukaemia. Blood Coagul Fibrinolysis, 5 Suppl 1, S24-36; discussion S59-64. doi:10.1097/00001721-199401000-00005

Caruso, V., Iacoviello, L., Di Castelnuovo, A., Storti, S., Mariani, G., de Gaetano, G., \& Donati, M. B. (2006). Thrombotic complications in childhood acute lymphoblastic leukemia: a meta-analysis of 17 prospective studies comprising 1752 pediatric patients. Blood, 108 (7), 2216-2222. doi:10.1182/blood-2006-04-015511

Douer, D., Yampolsky, H., Cohen, L. J., Watkins, K., Levine, A. M., Periclou, A. P., \& Avramis, V. I. (2007). Pharmacodynamics and safety of intravenous pegaspargase during remission induction in adults 
aged 55 years or younger with newly diagnosed acute lymphoblastic leukemia.Blood, 109 (7), 2744-2750. doi:10.1182/blood-2006-07-035006

Elhasid, R., Lanir, N., Sharon, R., Weyl Ben Arush, M., Levin, C., Postovsky, S., . . Brenner, B. (2001). Prophylactic therapy with enoxaparin during L-asparaginase treatment in children with acute lymphoblastic leukemia. Blood Coagul Fibrinolysis, 12 (5), 367-370. doi:10.1097/00001721-200107000-00005

Gupta, S., Wang, C., Raetz, E. A., Schore, R., Salzer, W. L., Larsen, E. C., . . Devidas, M. (2020). Impact of Asparaginase Discontinuation on Outcome in Childhood Acute Lymphoblastic Leukemia: A Report From the Children's Oncology Group. J Clin Oncol, 38 (17), 1897-1905. doi:10.1200/JCO.19.03024

Greiner J., Schrappe, M., Claviez, A., Zimmerman, M., Niemeyer, C., Kolb, R., (2019). THROMBOTECT a randomized study comparing low molecular weight heparin, antithrombin and unfractionated heparin fro thromboprophylaxis during induction therapy of acute lymphoblastic leukemia in children and adolescents. Haematologica ,104(4): 756-765 . doi: 10.3324/haematol.2018.194175

Harris, P. A., Taylor, R., Minor, B. L., Elliott, V., Fernandez, M., O’Neal, L., . . Consortium, R. E. (2019). The REDCap consortium: Building an international community of software platform partners.J Biomed Inform, 95 , 103208. doi:10.1016/j.jbi.2019.103208

Harris, P. A., Taylor, R., Thielke, R., Payne, J., Gonzalez, N., \& Conde, J. G. (2009). Research electronic data capture (REDCap)-a metadata-driven methodology and workflow process for providing translational research informatics support. J Biomed Inform, 42 (2), 377-381. doi:10.1016/j.jbi.2008.08.010

Hernandez-Espinosa, D., Minano, A., Martinez, C., Perez-Ceballos, E., Heras, I., Fuster, J. L., . . Corral, J. (2006). L-asparaginase-induced antithrombin type I deficiency: implications for conformational diseases. Am J Pathol, 169 (1), 142-153. doi:10.2353/ajpath.2006.051238

Hongo, T., Okada, S., Ohzeki, T., Ohta, H., Nishimura, S., Hamamoto, K., . . Ueda, K. (2002). Low plasma levels of hemostatic proteins during the induction phase in children with acute lymphoblastic leukemia: A retrospective study by the JACLS. Japan Association of Childhood Leukemia Study. Pediatr Int, 44 (3), 293-299. doi:10.1046/j.1442-200x.2002.01556.x

Kidd, J. G. (1953). Regression of transplanted lymphomas induced in vivo by means of normal guinea pig serum. II. Studies on the nature of the active serum constituent: histological mechanism of the regression: tests for effects of guinea pig serum on lymphoma cells in vitro: discussion. J Exp Med, 98 (6), 583-606. doi:10.1084/jem.98.6.583

Male, C., Lensing, A., Palumbo J., Kumar R., Nurmeev I., Hege K., .. (2020). Rivaroxaban compared with standard anticoagulants for the treatment of acute venous thromboembolism in children: a randomised, controlled, phase 3 trail. Lancet Haematol , 7(1): e18-e27.doi: 10.1016/S2352-3026(19)30219-4.

Meister, B., Kropshofer, G., Klein-Franke, A., Strasak, A. M., Hager, J., \& Streif, W. (2008). Comparison of low-molecular-weight heparin and antithrombin versus antithrombin alone for the prevention of symptomatic venous thromboembolism in children with acute lymphoblastic leukemia.Pediatr Blood Cancer, 50 (2), 298303. doi:10.1002/pbc. 21222

Mitchell, L., Andrew, M., Hanna, K., Abshire, T., Halton, J., Wu, J., . . . Mikulis, D. (2003). Trend to efficacy and safety using antithrombin concentrate in prevention of thrombosis in children receiving lasparaginase for acute lymphoblastic leukemia. Results of the PAARKA study. Thromb Haemost, 90 (2), 235-244. doi:10.1160/TH02-11-0283

Mitchell, L., Hoogendoorn, H., Giles, A. R., Vegh, P., \& Andrew, M. (1994). Increased endogenous thrombin generation in children with acute lymphoblastic leukemia: risk of thrombotic complications in L'Asparaginase-induced antithrombin III deficiency. Blood, 83 (2), 386-391. Retrieved from https://www.ncbi.nlm.nih.gov/pubmed/8286739 
Mitchell, L. G., Andrew, M., Hanna, K., Abshire, T., Halton, J., Anderson, R., . . Prophylactic Antithrombin Replacement in Kids with Acute Lymphoblastic Leukemia Treated with Asparaginase, G. (2003). A prospective cohort study determining the prevalence of thrombotic events in children with acute lymphoblastic leukemia and a central venous line who are treated with L-asparaginase: results of the Prophylactic Antithrombin Replacement in Kids with Acute Lymphoblastic Leukemia Treated with Asparaginase (PARKAA) Study. Cancer, 97 (2), 508-516. doi:10.1002/cncr.11042

Mitchell, L. G., Halton, J. M., Vegh, P. A., Barr, R. D., Venneri, T., Pai, K. M., \& Andrew, M. E. (1994). Effect of disease and chemotherapy on hemostasis in children with acute lymphoid leukemia. Am J Pediatr Hematol Oncol, 16 (2), 120-126. Retrieved from https://www.ncbi.nlm.nih.gov/pubmed/8166364

Monagle, P., Chan, A. K. C., Goldenberg, N. A., Ichord, R. N., Journeycake, J. M., Nowak-Gottl, U., \& Vesely, S. K. (2012). Antithrombotic therapy in neonates and children: Antithrombotic Therapy and Prevention of Thrombosis, 9th ed: American College of Chest Physicians Evidence-Based Clinical Practice Guidelines. Chest, 141 (2 Suppl), e737S-e801S. doi:10.1378/chest.11-2308

Monagle, P., Cuello, C. A., Augustine, C., Bonduel, M., Brandao, L. R., Capman, T., . . Vesely, S. K. (2018). American Society of Hematology 2018 Guidelines for management of venous thromboembolism: treatment of pediatric venous thromboembolism. Blood Adv, 2 (22), 3292-3316. doi:10.1182/bloodadvances.2018024786

Nowak-Gottl, U., Kenet, G., \& Mitchell, L. G. (2009). Thrombosis in childhood acute lymphoblastic leukaemia: epidemiology, aetiology, diagnosis, prevention and treatment. Best Pract Res Clin Haematol, 22 (1), 103-114. doi:10.1016/j.beha.2009.01.003

O'Brien, S. H., Li, D., Mitchell, L. G., Hess, T., Zee, P., Yee, D. L., . . Rodriguez, V. (2019). PREVAPIXALL: Apixaban Compared to Standard of Care for Prevention of Venous Thrombosis in Paediatric Acute Lymphoblastic Leukaemia (ALL)-Rationale and Design. Thromb Haemost, 119 (5), 844-853. doi:10.1055/s0039-1679938

Ortel, T. L., Neumann, I., Ageno, W., Beyth, R., Clark, N. P., Cuker, A., . . Zhang, Y. (2020). American Society of Hematology 2020 guidelines for management of venous thromboembolism: treatment of deep vein thrombosis and pulmonary embolism. Blood Adv, 4 (19), 4693-4738. doi:10.1182/bloodadvances.2020001830

Payne, J. H., \& Vora, A. J. (2007). Thrombosis and acute lymphoblastic leukaemia. Br J Haematol, 138 (4), 430-445. doi:10.1111/j.1365-2141.2007.06677.x

Plourde, P. V., Jeha, S., Hijiya, N., Keller, F. G., Silverman, L. B., Rheingold, S. R., . . Corn, T. (2014). Safety profile of asparaginase Erwinia chrysanthemi in a large compassionate-use trial. Pediatr Blood Cancer, 61 (7), 1232-1238. doi:10.1002/pbc.24938

Qubty, W. F., Mrelashvili, A., \& Patterson, M. C. (2015). Epidural hematoma in a patient on pegylated-Lasparginase therapy. J Child Neurol, 30 (5), 636. doi:10.1177/0883073813512900

Qureshi, A., Mitchell, C., Richards, S., Vora, A., \& Goulden, N. (2010). Asparaginase-related venous thrombosis in UKALL 2003- re-exposure to asparaginase is feasible and safe. Br J Haematol, 149 (3), 410-413. doi:10.1111/j.1365-2141.2010.08132.x

Salzer, W. L., Asselin, B., Supko, J. G., Devidas, M., Kaiser, N. A., Plourde, P., . . Hunger, S. P. (2013). Erwinia asparaginase achieves therapeutic activity after pegaspargase allergy: a report from the Children's Oncology Group. Blood, 122 (4), 507-514. doi:10.1182/blood-2013-01-480822

Stock, W., Douer, D., DeAngelo, D. J., Arellano, M., Advani, A., Damon, L., . . Bleyer, A. (2011). Prevention and management of asparaginase/pegasparaginase-associated toxicities in adults and older adolescents: recommendations of an expert panel. Leuk Lymphoma, 52 (12), 2237-2253. doi:10.3109/10428194.2011.596963

\section{FIGURE LEGEND}


FIGURE 1. Fibrinogen Cut off and Replacement Goal . $74.5 \%(\mathrm{n}=91 / 122)$ of respondents use $100 \mathrm{mg} / \mathrm{dL}$ as cut-off level to consider fibrinogen replacement, which is reflected in this box plot. Dots represent outliers. The horizontal line represents the median. X represents the mean.

FIGURE 2. Antithrombin Cut Off and Replacement Goals . Boxes represent interquartile range and whiskers represent minimum and maximum values that respondents use as antithrombin cut offs and replacement goals. Dots represent outliers. The horizontal line represents the median. X represents the mean.

Table 1: Sample Characteristics of Respondents

\section{Hosted file}

Table 1 Sample Characteristics Peg Asparaginase in Pediatric Patients.docx available at https://authorea. com/users/426458/articles/530984-management-practices-for-asparaginaseassociated-coagulopathy-a-survey-of-pediatric-oncologists

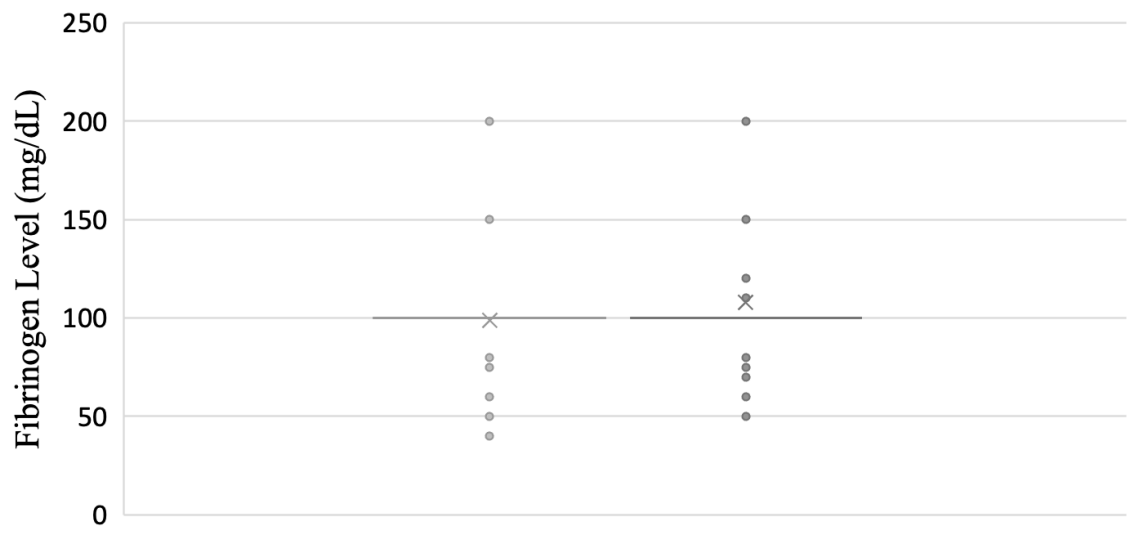

$\square$ Fibrinogen Replacement Trigger $\square$ Fibrinogen Replacement Goal

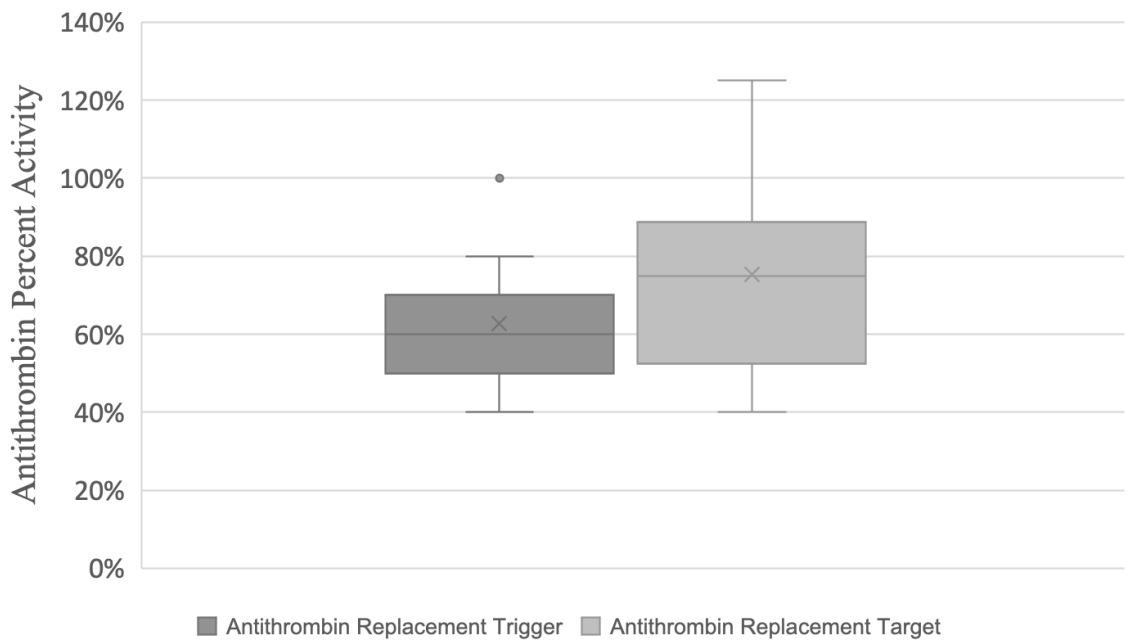

